\title{
Publisher's Note: Logic gates with bright dissipative polariton solitons in Bragg cavity systems [Phys. Rev. B 92, 174528 (2015)]
}

\author{
E. Cancellieri, J. K. Chana, M. Sich, D. N. Krizhanovskii, M. S. Skolnick, and D. M. Whittaker
}

(Received 2 February 2017; published 10 February 2017)

DOI: 10.1103/PhysRevB.95.059902

This paper was published online on 24 November 2015 with missing information in the Acknowledgments. The Acknowledgments should read as "We acknowledge support by EPSRC (UK) Grant No. EP/J007544/1, ERC Advanced Grant No. EXCIPOL 320570, and Leverhulme Trust Grant No. RPG-2013-339." The Acknowledgments have been corrected as of 2 February 2017. The Acknowledgments are incorrect in the printed version of the journal. 